\title{
Seehaus, Rhea
}

\section{(Un-)Ordnungen der familialen Sorge? Mütter und die diskursive Figur des ,aktiven Vaters“}

Dausien, Bettina [Hrsg.]; Thon, Christine [Hrsg.]; Walgenbach, Katharina [Hrsg.]: Geschlecht - Sozialisation

- Transformationen. Opladen ; Berlin ; Toronto : Verlag Barbara Budrich 2015, S. 163-178. - (Jahrbuch

Frauen- und Geschlechterforschung in der Erziehungswissenschaft; 11)

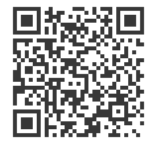

Quellenangabe/ Reference:

Seehaus, Rhea: (Un-)Ordnungen der familialen Sorge? Mütter und die diskursive Figur des ,aktiven Vaters' - In: Dausien, Bettina [Hrsg.]; Thon, Christine [Hrsg.]; Walgenbach, Katharina [Hrsg.]:

Geschlecht - Sozialisation - Transformationen. Opladen ; Berlin ; Toronto : Verlag Barbara Budrich 2015, S. 163-178 - URN: urn:nbn:de:0111-pedocs-130375 - DOI: 10.25656/01:13037

https://nbn-resolving.org/urn:nbn:de:0111-pedocs-130375

https://doi.org/10.25656/01:13037

in Kooperation mit / in cooperation with:

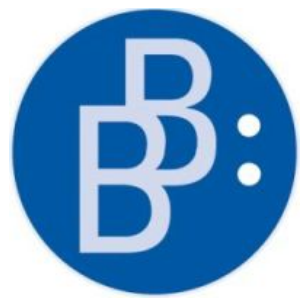

https://www.budrich.de

\section{Nutzungsbedingungen}

Gewährt wird ein nicht exklusives, nicht übertragbares, persönliches und beschränktes Recht auf Nutzung dieses Dokuments. Dieses Dokument ist ausschließlich für den persönlichen, nicht-kommerziellen Gebrauch bestimmt. Die Nutzung stellt keine Übertragung des Eigentumsrechts an diesem Dokument dar und gilt vorbehaltlich der folgenden Einschränkungen: Auf sämtlichen Kopien dieses Dokuments müssen alle Urheberrechtshinweise und sonstigen Hinweise auf gesetzlichen Schutz beibehalten werden. Sie dürfen dieses Dokument nicht in irgendeiner Weise abändern, noch dürfen Sie dieses Dokument für öffentliche oder kommerzielle Zwecke vervielfältigen, öffentlich ausstellen, aufführen, vertreiben oder anderweitig nutzen.

Mit der Verwendung dieses Dokuments erkennen Sie die Nutzungsbedingungen an.

\section{Terms of use}

We grant a non-exclusive, non-transferable, individual and limited right to using this document.

This document is solely intended for your personal, non-commercial use. Use of this document does not include any transfer of property rights and it is conditional to the following limitations: All of the copies of this documents must retain all copyright information and other information regarding legal protection. You are not allowed to alter this document in any way, to copy it for public or commercial purposes, to exhibit the document in public, to perform, distribute or otherwise use the document in public.

By using this particular document, you accept the above-stated conditions of use.

\section{Kontakt / Contact:}

\section{peDOCS}

DIPF | Leibniz-Institut für Bildungsforschung und Bildungsinformation

Informationszentrum (IZ) Bildung

E-Mail: pedocs@dipf.de

Internet: www.pedocs.de

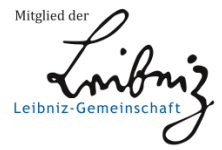


Bettina Dausien
Christine Thon
Katharina Walgenbach (Hrsg.)

11/2015

Jahrbuch Frauen- und Geschlechterforschung in der Erziehungswissenschaft

Geschlecht - Sozialisation - Transformationen 
Jahrbuch

Frauen- und Geschlechterforschung in der Erziehungswissenschaft

Redaktion

Jürgen Budde

Astrid Messerschmidt

Christine Thon

Katharina Walgenbach

Beirat

Birgit Althans

Sabine Andresen

Eva Breitenbach

Rita Casale

Bettina Dausien

Isabell Diehm

Hannelore Faulstich-Wieland

Edgar Forster

Edith Glaser

Carola Iller

Marita Kampshoff

Margret Kraul

Andrea Liesner

Susanne Maurer

Vera Moser

Inga Pinhard

Annedore Prengel

Barbara Rendtorff

Folge 11/2015 
Bettina Dausien

Christine Thon

Katharina Walgenbach (Hrsg.)

\section{Geschlecht - Sozialisation - Transformationen}

Verlag Barbara Budrich

Opladen • Berlin • Toronto 2015 
Bibliografische Information der Deutschen Nationalbibliothek

Die Deutsche Nationalbibliothek verzeichnet diese Publikation in der Deutschen Nationalbibliografie; detaillierte bibliografische Daten sind im Internet über

http://dnb.d-nb.de abrufbar.

Gedruckt auf säurefreiem und alterungsbeständigem Papier.

Alle Rechte vorbehalten.

C) 2015 Verlag Barbara Budrich, Opladen, Berlin \& Toronto

www.budrich-verlag.de

ISBN 978-3-8474-0668-6 (Paperback)

eISBN 978-3-8474-0854-3 (eBook)

Das Werk einschließlich aller seiner Teile ist urheberrechtlich geschützt. Jede Verwertung außerhalb der engen Grenzen des Urheberrechtsgesetzes ist ohne Zustimmung des Verlages unzulässig und strafbar. Das gilt insbesondere für Vervielfältigungen, Übersetzungen, Mikroverfilmungen und die Einspeicherung und Verarbeitung in elektronischen Systemen.

Umschlaggestaltung: disegno visuelle kommunikation, Wuppertal - www.disenjo.de Lektorat: Christine Berberich

Typographisches Lektorat: Ulrike Weingärtner, Gründau 


\section{Inhalt}

\section{Einleitung}

Bettina Dausien, Christine Thon, Katharina Walgenbach

Geschlecht - Sozialisation - Transformationen

\section{Essay}

Bettina Dausien, Katharina Walgenbach

Sozialisation von Geschlecht -

Skizzen zu einem wissenschaftlichen Diskurs und Plädoyer für

die Revitalisierung einer gesellschaftsanalytischen Perspektive

\section{Theoretische Analysen - Transformationen von Debatten}

\section{Jutta Hartmann}

Transformatorische Denkbewegungen -

Weiterentwicklung von Sozialisationstheorien im Anschluss an

Judith Butlers diskurstheoretisches Verständnis von Geschlecht

Barbara Rendtorff

Zugewinne und Fallen - aktuelle Veränderungen

in Geschlechtervorstellungen und ihre Probleme

Mechthild Bereswill, Gudrun Ehlert

Sozialisation im Kontext des Krisendiskurses über Jungen

Empirische Analysen -

Transformationen von Geschlechterverhältnissen

Sabine Klinger

Die (De-)Thematisierung von Geschlechterhierarchien im Verhältnis akademischer Sozialisationsprozesse und gesellschaftlicher Diskurse.

Eine qualitativ-rekonstruktive Analyse studentischer

Gruppendiskussionen

Monika Götsch

Paradoxien heteronormativer Sozialisation 
Inhalt

\section{Offener Teil}

Karin Flaake

Neue Konstellationen für Männlichkeitsentwürfe -

Potentiale einer in der Paarbeziehung geteilten Elternschaft

für Entwicklungsmöglichkeiten von Jungen und jungen Männern

Rhea Seehaus

(Un-)Ordnungen der familialen Sorge?

Mütter und die diskursive Figur des , aktiven Vaters ${ }^{6}$

Stefanie Leinfellner

Ambivalenzen und transformative Potenziale im Arbeitskontext

Wissenschaft: Zur Vereinbarkeit von Wissenschaft und Elternschaft in Dual-Career-Familien

\section{Tagungsbericht}

Anne-Dorothee Warmuth

„Erziehung - Gewalt - Sexualität“. Tagungsbericht zur Jahrestagung der Sektion Frauen- und Geschlechterforschung der DGfE an der Universität Paderborn (05./06. März 2015)

Verzeichnis der Autor_innen 209 


\title{
(Un-)Ordnungen der familialen Sorge? Mütter und die diskursive Figur des , aktiven Vaters ${ }^{6}$
}

\author{
Rhea Seehaus
}

\section{Einleitung}

Für die Geschlechterverhältnisse der familialen Sorgearbeit lassen sich gegenwärtig diskursive und praktische Transformationsprozesse festhalten. Sowohl wissenschaftliche Studien als auch öffentliche Diskussionen verweisen darauf, dass die väterliche Beteiligung am Schwangerschaftsgeschehen und bei der Geburt sowie die spätere Involvierung in die elterliche Sorgearbeit zugenommen haben (vgl. u.a. Otto 2011) und - nicht zuletzt über die ,Vätermonate' des Elterngeldes - auch staatlich gefördert werden (vgl. u.a. Ehnis/Beckmann 2010). Familiale Sorge-Aufgaben, so präsentieren es Politik und Medien, sollen nun zwischen den Eltern aufgeteilt werden und nicht mehr ausschließlich in den Zuständigkeitsbereich von Müttern fallen. Bisherige Geschlechtertexte in der Elternschaft scheinen vielfach in Auflösung begriffen zu sein (vgl. Jurczyk/ Thiessen 2011: 339), bzw. werden plötzlich zum Aushandlungsgegenstand öffentlichen Interesses - festgemacht wird dies immer wieder an der Figur des ,aktiven Vaters'. Diese auf die Sorgearbeit bezogenen Umbrüche in den Vaterschaftskonzepten und -praxen werden in den Sozialwissenschaften relativ intensiv thematisiert, vaterbezogene Forschung mit dem Schwerpunkt des , aktiven Vaters' ist in den letzten Jahren deutlich expandiert (vgl. u.a. Ehnis 2009; Possinger 2013). Aus dem (wissenschaftlichen) Blick gerät jedoch vielfach das Verhältnis der Mütter zu dieser neuen diskursiven Orientierungsfigur. Diesem soll im Folgenden anhand der Ergebnisse einer Interviewstudie nachgegangen werden. 


\section{Die familiale Sorge um das Kind - aktive Vaterschaft als historisch neues Phänomen}

Sorgearbeit findet in vielfältigen Bereichen des sozialen Lebens statt, der vorliegende Beitrag fokussiert sie im Bereich von Elternschaft. ${ }^{1}$ Aus der Geburt eines Kindes resultiert für Eltern die Notwendigkeit, Sorgearbeit zu leisten. Aufgrund der leiblichen Angewiesenheit des Säuglings muss der familiale Alltag (neu) organisiert und ausgehandelt werden: Gebürtlichkeit wird damit analog zur Sterblichkeit - zum konstituierenden Element von Sorgearbeit. Wie diese organisiert ist, ob sie vorrangig als familiale Aufgabe gesehen wird oder durch professionelle Dienstleistungen ergänzt wird, kann - je nach gesellschaftlicher Verfasstheit - unterschiedlich geregelt werden und lässt sich auch nicht von historischen Entwicklungen trennen. Fragen des Sorgens und Entwürfe der Frauenrolle scheinen jedoch untrennbar verwoben (vgl. Moser/Pinhard 2010: 11) - darauf haben nicht zuletzt auch die jeweiligen wohlfahrtsstaatlichen Regelungen und hegemonialen Diskurse sowie die interaktiven Praxen ,im Kleinen“ einen nicht unerheblichen Einfluss. Jurczyk und Rerrich merken dazu an: „Durch gezielte normative Regelungen wie auch , unter der Hand' wird durch soziale Zuweisungs- und Codierungsprozesse eine bestimmte Art der Lebensführung, die die praktische Sorge für andere Personen umfasst, mit ihren Elementen der Angebundenheit und Emotionalität, zur typisch weiblichen Lebensführung gemacht" (1993: 274).

Für Elternschaft lässt sich somit eine enge Verknüpfung von Generationenund Geschlechterordnung konstatieren, wenngleich mittlerweile immer wieder die Entstehung neuer ,Ordnungen der Sorge' (vgl. Toppe 2010) konstatiert wird, in denen sich neue (normative) Elternschafts- und Geschlechterideale abzeichnen. Diese rücken insbesondere den Vater ins Zentrum der Aufmerksamkeit: Bilder des , aktiven Vaters' sind in Werbung und Alltag zunehmend vertreten und positiv konnotiert (vgl. u.a. Baader 2006: 117; Thiessen/Villa 2009: 7). ${ }^{2}$ Allmählich bilden sich gesellschaftliche Orientierungsmuster für neue Vaterschaftsentwürfe heraus und dem aktiven Vater werden in zunehmendem Maße Anerkennung und Achtung gezollt: Er wird nicht mehr ausschließlich

1 Sorge wird im Sinne von care als interaktive Praxis verstanden, die auf Zeit, kommunikativen Raum und materielle Ressourcen angewiesen ist. Unter dem Begriff care lässt sich demnach „eine Vielfalt von reproduktiven, fürsorglichen, betreuenden und pflegenden Tätigkeiten“ (Gerhard 2010: 221) fassen. Er schließt an die in den 1970er und 80er Jahren von feministischen Auseinandersetzungen angestoßenen Diskussionen zum Thema Haus- und Familienarbeit an (vgl. Gather/Othmer/Senghaas-Knobloch 2013: 204).

2 Diese Studien sowie die folgenden beziehen sich in der Regel auf heterosexuelle (verheiratete) Eltern mit eigenen Kindern. Dies bedeutet eine starke Fokussierung auf eine spezielle Familienform, die andere Familienformen nicht in den Blick nimmt. Dies ist jedoch zugleich auch die Familienform, die im Sample der vorliegenden Studie repräsentiert ist. Siehe Anmerkung 5 . 
auf die „Alimentation des Nachwuchses“(Jurczyk/Lange 2009: 14) verpflichtet, sondern die familiale Sorgearbeit soll geteilt werden. Väter sollen eine emotional-zugewandte Bindung zum Säugling und Kind entwickeln und sich verantwortlich kümmern (vgl. u.a. Ehnis/Beckmann 2010: 313ff.). Das ,fathering“ (Meuser 2009: 220) ist in höchstem Maße erwünscht, betont wird mittlerweile auch die Bedeutung der fürsorglichen Väter für die kindliche Entwicklung und Sozialisation (vgl. Reinwand 2012: 431f.). Im Zuge dieser Entwicklungen sind Forschungen zum Thema Vaterschaft deutlich expandiert: Gefragt wird beispielsweise nach neuen (diskursiven) Männlichkeitsentwürfen im Kontext von Vaterschaft (u.a. Kassner 2008; Wolde 2007), subjektiven Vaterschaftskonzepten (u.a. Matzner 2004) sowie Vaterschaft und Erwerbsarbeit (u.a. Ehnis 2009; Possinger 2013). Die Studien zeigen nachdrücklich, dass die Figur des Vaters als Haupternährer allmählich brüchig wird und sich nicht nur neue Familienbilder, sondern auch neue, pluralisierte Vaterschaftsformen entwickeln, in denen der, aktive Vater ${ }^{63}$ zum Leitbild avanciert. Studien zur Arbeitsteilung (vgl. Kortendiek 2010: 446) zeigen jedoch auch, dass sich, obwohl mit der Figur des aktiven Vaters zugleich auch egalitäre Elternschaft zur neuen familialen Norm wird (vgl. Correll 2010: 270) und sich diskursiv die ,Ordnungen der Sorge' verändern, nicht zwangsläufig entsprechend tragfähige Elternschaftsrealitäten ausbilden. Vielen Elternpaaren gelingt es offenbar trotz guter Absichten nur unzureichend, den gewünschten Weg zum paritätischen ElternSein auch erfolgreich zu gehen: Die pränatalen Selbstansprüche egalitärer Elternschaft sind nach der Geburt offenbar nur sehr schwer tatsächlich (langfristig) zu realisieren (vgl. Rüling 2010: 4775). Palz, Werneck und Beham (2006: 15) verweisen darauf, dass der Prozentsatz derjenigen Väter, die als aktive zu bezeichnen seien, vor der Geburt des Kindes weitaus höher lag als drei Jahre nach der Geburt. Auch Kassner (2008: 143) weist in seiner Untersuchung darauf hin, dass die Erosion normativer Leitbilder nicht zwangsläufig eine veränderte Vaterschafts- bzw. Familienpraxis bedeutet. Veränderungen bezögen sich vielfach nur auf die Ebene der Diskurse und Leitbilder und weniger auf die Ebenen kollektiver Deutungsmuster und individueller Praxen.

Die in Familien stattfindenden Entwicklungen lassen sich als Re-Traditionalisierungseffekte bezeichnen (vgl. u.a. die Studien von Reichle 1996; Rüling 2007; Walter/Künzel 2002), die als ,besonderes Moment der Aktivierung alter Muster mit dem Elternwerden" (Kerschgens 2009: 18) beschrieben werden können. Elternschaft und die potentiell stattfindenden, auf die Geschlechterverhältnisse bezogenen Transformationsprozesse werden vielfach ausschließlich über die vaterschaftsbezogenen Veränderungen wissenschaftlich in den Blick genommen. Eine ähnliche Konjunktur bei der Mutterschaftsforschung lässt sich nicht ausmachen. Dabei verändert sich, z.B. aufgrund der „Norm der 
Doppelverdienerfamilie“ (Fraser 2009: 51), die zu einer allgemeinen Erwerbsarbeitszentrierung des Lebens sowohl von Männern als auch von Frauen führt (vgl. König 2012: 48), vermutlich auch das Leben von Müttern. Ob und was dies für die mütterliche Sorgearbeit bedeutet, bleibt in der Regel unerwähnt. Hünig und Peter bemerken dazu, dass explizite „Mütterbelange und Problemlagen von Frauen" (2010: 191f.) meistens nur "implizit im Kontext von Familienrhetorik (mit-)verhandelt und diskutiert“ (ebd.) und deshalb im „erziehungswissenschaftlichen Fachdiskurs fehlen“" (ebd. 192) würden.

\section{Fragestellung und Methode}

Vor dem Hintergrund eines abgeschlossenen qualitativen Forschungsprojekts zur elterlichen Sorge um das Kind (Seehaus 2014) ${ }^{4}$ wird im Folgenden stärker die Perspektive der Mütter ins Zentrum gerückt. Ausgangspunkt der Analysen ist die Beobachtung, dass in den Interviews, obwohl in den meisten Familien die Mütter als die Hauptzuständigen für die Sorgearbeit dargestellt werden, insbesondere von ihnen das Engagement der Väter sehr ausführlich thematisiert wird (vgl. Seehaus 2014: 59f.). Im Sinne der thematischen Diskursanalyse (Höhne 2003) wird das Interview als Instanz der Diskursreproduktion verstanden und die Darstellungen der Mütter bzw. Eltern zur innerfamilialen Arbeitsteilung werden als Ausgangspunkt genutzt, um zu untersuchen, wie geschlechtsspezifische Zuständigkeiten thematisiert werden und wie in diesen zugleich mögliche Anrufungen und Aufforderungen durch Diskurse, gesellschaftliche Normen und eigene Vorstellungen sichtbar werden - in aller Widersprüchlichkeit, die diese mit sich bringen. Dabei wird die These vertreten, dass die Figur des aktiven Vaters, die in aktuellen Elternschafts- und Vaterschaftsdiskursen so prominent ist, gerade nicht nur Väter anruft, sich als solche

4 Die Studie untersucht anhand von Interviews mit Müttern, Vätern und Eltern die Konstruktionslogiken von Elternverantwortung. Fokussiert werden dabei die normativen Rahmungen dieser ,Sorge um das Kind' und ihre Folgen für Geschlechterverhältnisse und Normierung von Elternschaft. Der offene Leitfaden enthielt die Themenkomplexe Familie(nleben), Entwicklung des Kindes, kindermedizinische Vorsorge-Untersuchungen und Elternbildung-/Elterninformationen. Es wurden 28 Interviews geführt, in 25 Familien waren die Kinder unter sechs Jahren, in drei Familien unter neun Jahren. In sechs Familien waren die Mütter zum Zeitpunkt des Interviews ausschließlich für die Sorgearbeit zuständig und gingen aus diesem Grund seinerzeit keiner Erwerbsarbeit nach. In drei Familien war mindestens ein Elternteil zum Interviewzeitpunkt gerade erwerbsarbeitslos. In fünf Familien hatten die Väter ihre Erwerbsarbeitszeit zugunsten der Sorgearbeit eingeschränkt. Das Sample weist eine relativ große soziale Homogenität auf, es sind überwiegend Eltern der ,Mittel- und Oberschicht". Dies bringt einerseits eine hohe Vergleichbarkeit der einzelnen Interviewausschnitte mit sich, während andererseits damit auch die Reichweite der Ergebnisse beschränkt ist: Die Figur des , aktiven Vaters' ist sicherlich nicht für alle Schichten in dieser Weise zentral (vgl. auch Meuser/Scholz 2012: 37). 
zu zeigen, sondern auch Mütter zu speziellen Selbstpositionierungen und Fremdpositionierungen bezüglich des Vaters herausfordert. Das Interview wird damit als Ort verstanden, an dem solche Anrufungen aktualisiert werden. Dies bedeutet zugleich auch, dass nicht die praktische Ausgestaltung der Elternschaft oder mögliche Widersprüche zwischen elterlichen Planungen und Praxen rekonstruiert werden. Ein solcher Anspruch auf eine egalitäre Ausgestaltung von Elternschaft wurde auch nicht erhoben. Aus diesem Grund wird auch im Folgenden keine weitere Differenzierung des Konzepts , aktiver Vater vorgenommen, wie sie Kassner (2008: 144) vorschlägt, um zwischen ,tatsächlich` egalitär verteilter Sorgearbeit und deutlich weniger egalitären Verteilungen zu differenzieren. Der , aktive Vater" wird in dem vorliegenden Beitrag nicht als spezieller Vatertypus in den Blick genommen, dessen Ausprägung, Verbreitung und Differenzierung untersucht werden sollen. Stattdessen soll er als diskursive Figur verstanden werden, auf die die InterviewpartnerInnen Bezug nehmen (müssen). Damit wird rekonstruiert, wie „Diskurse an Subjekte gelangen" (Correll 2010: 80), und insbesondere auf die Mütter fokussiert.

Um dies zu rekonstruieren, greife ich auf das Konzept der, Anrufung' (vgl. u.a. Althusser 1977; Butler 1997) als sensibilisierendes Konzept der Analyse zurück. Anrufungen sind spezielle Adressierungen der Individuen als Subjekte, die sich als „,soziale Zuschreibungen“ verstehen lassen, „die die Aufforderungen zu bestimmten Verhaltens- und (Be-)Handlungsweisen vermitteln“ (Ott/Wrana 2014: 19). Solche Adressierungen werden durch institutionalisierte Settings vollzogen oder sind diskursiv in gesellschaftlichen Institutionen, politischen Programmen und sozialen Konstellationen enthalten (vgl. ebd.). Anrufungen können demnach als Adressierungen durch eine spezifische Ordnung verstanden werden, auf die die Individuen reagieren (vgl. Wrana 2006: 136). Konstitutiv für das ,Funktionieren“ der Anrufung ist jedoch nicht nur die Adressierung, sondern auch ihre Beantwortung (vgl. Graefe 2010: 292): Die Subjekte müssen diese Subjektpositionen selbstständig unter Ausnutzung ihres Handlungsspielraums ausfüllen, was bedeutet, dass sie sich den Anrufungen nicht einfach , unterwerfen', sondern sich diese eigensinnig aneignen - den Subjekten ist es durchaus möglich, „dem Ruf nicht in der erwarteten Weise zu folgen" (Graefe 2010: 292).

Diese zweite, antwortende Seite der Anrufungen ist diejenige, welche im Folgenden betrachtet werden soll. Ich setze deshalb bei den auf die väterliche Sorge und den aktiven Vater bezogenen Äußerungen ${ }^{5}$ an und rekonstruiere, „was in den Redeereignissen des Interviews“ (König 2008: 4789) geschieht und welche Selbst- und Machtverhältnisse in der „Logik dieses Geschehens“ (ebd.) konstituiert werden.

5 Diese Szenen wurden aus dem Gesamtmaterial der Studie mithilfe einer thematischen Kodierung markiert. 


\section{Neu-Ordnungen der Sorge - empirische Ergebnisse}

Während zwar einerseits in den Interviews die Sorgearbeit beständig feminisiert und zur Hauptaufgabe der Mutter erklärt wird, ist andererseits oftmals das väterliche Engagement Gegenstand der Narrationen der Mütter - der , aktive Vater' wird zur Leitfigur. Die Bezugnahmen auf diese Figur können zu zwei Thematisierungsweisen verdichtet werden, die hier anhand von Interviewsequenzen exemplarisch illustriert werden sollen.

\subsection{Verhinderte Väter-Zeitkonkurrenzen aktiver Väter}

In einem Teil der auf den Vater bezogenen Interviewsequenzen wird insbesondere das Thema Zeit in den Vordergrund gerückt. Während das Zeitbudget der Mütter offenbar eines ist, das in der Wahrnehmung der InterviewpartnerInnen nur selten konkurrierende Zeiten enthält und deshalb kaum Erwähnung findet (vgl. Seehaus 2014: 97), stellen die Mütter immer wieder besondere Zeitkonkurrenzen der Väter heraus, die sich in einer Unzufriedenheit der Väter niederschlagen: Diese würden gerne mehr Zeit mit den Kindern verbringen, aufgrund der Konkurrenz zwischen Erwerbs- und Sorgezeit sei dies jedoch nur schwer zu bewerkstelligen. Thematisiert werden vielfältige familiale Praktiken, mit denen versucht werde, dem Vater mehr Zeit mit dem Kind zu ermöglichen, so zum Beispiel ein sehr früher Arbeitsbeginn, die Parzellierung von Arbeitszeiten oder die gemeinsam verbrachte Mittagspause. Der aktive Vater, um den es in den Darstellungen letztendlich immer geht, wird als , verhinderter', in seiner Sorgezeit von externen Einflüssen eingeschränkter Vater entworfen. Interessanterweise zeigt sich in den Interviewsequenzen, dass diese Zeitkonkurrenzen weniger mit der väterlichen Erwerbsarbeit begründet als vielmehr durch die individuellen Zeitrhythmen des Kindes verursacht dargestellt werden, so zum Beispiel, dass die Schlafenszeit des Kindes immer mit dem Heimkommen des Vaters am Abend zusammenfalle. Nicht die väterliche Erwerbszeit, sondern die ,Kinderzeit' macht Probleme.

Anhand der folgenden Sequenz soll gezeigt werden, dass jedoch nicht nur die Sorge- und Erwerbsarbeit miteinander konkurrieren können, sondern mitunter auch die Freizeit. Zugleich rückt damit auch die diskursive Dynamik in den Blick, die im Interview entstehen kann, wenn dem Vater kein sehr großes Engagement bescheinigt wird. Die Interviewerin stellt der Mutter die Frage, ob sie als Paar vor der Geburt des Kindes bestimmte Vorstellungen hatten, wie das Familienleben aussehen könnte. Die Interviewte berichtet ausführlich über 
den Wunsch der Eltern, dass die Mutter eine möglichst lange Elternzeit nehmen würde. Daraufhin setzt folgende Sequenz ein ${ }^{6}$ :

Fr. W.: Für mich war's eigentlich eh klar, weil ich mir gedacht hab, ja wenn ich in Karenz bin, dann bin ich sowieso immer beim Baby und und für für nen Papa eben da war's, (...) bin ich auch erst nachher eigentlich draufkommen, dass er also schon $\mathrm{mhm}$ (..) er hat sich für sich selber eigentlich so (..) glaub ich des so vorgestellt dass er dann viel mehr (.) Zeit mit uns verbringt. Also so am Abend, nach dem Arbeiten und und halt nur, (.) was weiß ich, ausnahmsweise mhm sich mit Freunden trifft oder halt so die Hobbies weiterführt und (..) das hat genau drei Wochen@geklappt@und dann ((Lachen)), dann hat er einfach auch gemerkt, so das=das haltet er irgendwie auch nicht aus. Und dann haben wir uns mal ausgesprochen und dann mhm ich mein, dass, es soll schon das bisherige Leben eigentlich irgendwie weiter geführt werden, also es soll sich net alles ändern, nur das, das ist dann einfach eh schon zu viel. (.) Es ändert sich sowieso viel ((Lachen)) ja und das war vielleicht das, ja was man sich vorher anders vorgestellt hatte.

(Interview Frau Wagner, S. 5, Z. 127-139)

Zwar wird das Zeitkonzept der Mutter als dasjenige dargestellt, welches nach der Geburt ausschließlich als Sorgezeit veranschlagt wird, jedoch werden auch für das Zeitkonzept des Vaters Veränderungen benannt. Während die Erwerbszeit nicht angetastet wird, hätte der Vater, so die Interviewte, seine Freizeit durch Sorgezeit ersetzen wollen. Diese versuchte Priorisierung konkurrierender Zeiten sei jedoch gescheitert, weil der Vater sie nicht ertragen hätte. Das ,Geständnis‘ der Mutter über dieses Scheitern wird lachend vorgetragen, so als solle die Nichteinhaltung der ursprünglich stärker egalitären Vorstellungen abgemildert werden. Möglicherweise ist dies auch einer Scham der Interviewerin gegenüber geschuldet, da eingestanden werden muss, dass der Vater zwar auf die aktuellen Adressierungen als aktiver Vater reagiert, jedoch nach kurzer Zeit die Freizeit vor der mit dem Kind verbrachten Zeit priorisiert. Die väterliche Sorgezeit wird in dieser Thematisierung - im Gegensatz zur mütterlichen Zeit - als fragil dargestellt: Sie bleibt Verhandlungssache und letztendlich rücknehmbar, auch wenn vorab andere Vorstellungen und Wünsche herrschten.

Betrachtet man den weiteren Interviewverlauf, so ist festzustellen, dass diese von der Mutter vorgenommene Positionierung des Vaters als wenig aktiver Vater von ihr nur eine Sequenz später deutlich abgeschwächt wird, als sie herausstellt, dass er die Subjektposition des aktiven Vaters doch übernehme.

Fr. W.: Ja, aber Hobbies hat er sehr (.) also bei seinen, (.) also er hat die ganze Zeit nen Hobby gehabt und das hat er jetzt eigentlich momentan ziemlich aufgegeben. Aber auch (.) er hat's (.) ja mhm auch leicht aufgeben. Es war nicht so, dass er gesagt hat:

6 Für die Transkription gelten folgende Regeln: (.) = kurze Gesprächspause (Anzahl der Punkte zeigt die Länge an), fett = laut gesprochen, unterstrichen = betont gesprochen, @ = lachend gesprochen. Alle Daten zu Personen, Orten und Berufsbezeichnungen wurden anonymisiert. 
„Okay, jetzt hab ich ein Baby und jetzt (spricht mit etwas weinerlicher, verstellter Stimme) muss ich halt", sondern „Es ist so, jetzt hat einfach sie hat jetzt einfach Priorität halt und das ist, (.) das passt da.“

(Interview Frau Wagner, S. 7, Z. 175-181)

Der vorherige Entwurf des Vaters als stark freizeitorientierter Vater, der wenig Zeit für sein Kind hat, wird mit Nachdruck relativiert: Der Vater hätte auf ein Hobby verzichtet und das sei ihm nicht schwergefallen. Damit wird ihm in dieser Thematisierung noch nachträglich die Übernahme der Subjektposition des aktiven Vaters attestiert. Insofern weist jedoch die Darstellung des väterlichen Engagements im Interviewverlauf keinen stringenten Verlauf auf: Mal wird sein (zeitliches) Engagement als eher gering ausgewiesen, an anderer Stelle wird dies jedoch abgeschwächt - so als müsste ein Ausgleich geschaffen werden. Dies lässt sich auch darauf zurückführen, dass die derzeitigen Diskurse um aktive Vaterschaft und egalitäre Elternschaft, in denen die Sorgearbeit zwischen den Elternteilen verteilt wird, vermutlich eine gewisse Wirksamkeit entfalten. Im Kontext des Interviews scheint es für die Mutter nicht einfach zu gestehen, dass der Vater sich entgegen seiner ursprünglichen Idee weitestgehend aus der Sorgearbeit zurückgezogen hat und ihr Arbeitsteilungsmodell einer geschlechterspezifischen Logik folgt. Stattdessen werden bestimmte Bereiche des väterlichen Engagements herausgestellt, um aufzuzeigen, inwiefern die Subjektposition des , aktiven' und damit auch des , guten 'Vaters ausgefüllt wird. Anzunehmen ist, dass diese typische Ambivalenz in den mütterlichen Erzählungen Konfliktpotentiale in der Elternbeziehung andeutet - auch wenn sie nicht explizit angesprochen werden.

\subsection{Anzuleitende Väter-Inkompetenzen aktiver Väter}

Wie bereits angedeutet, zeigt sich in vielen Interviews mit Müttern ein doppelter Bezug auf das väterliche Engagement. Zwar wird einerseits an die Subjektposition des aktiven Vaters angeknüpft und diese für den eigenen Partner beansprucht, andererseits erfolgt häufig auch eine Relationierung des väterlichen Engagements mit dem mütterlichen, bei der letztendlich die väterliche Subjektivierung hinsichtlich der aktiven Vaterschaft angezweifelt wird. Diese zweite Thematisierungsweise soll im Folgenden exemplarisch an einem Auszug aus einem Paarinterview beleuchtet werden. In der Sequenz wird der Interviewerin zu Beginn ein gleichberechtigtes Elternpaar präsentiert, das sich dagegen wehrt, Mütter per se als die kompetenteren Elternteile im Bereich der Sorgearbeit zu verstehen, während dies im Interviewverlauf auf interaktiver Ebene in gegenteiliger Weise ausgestaltet wird. Die Interviewstelle ist Teil einer längeren Passage, in der die Interviewerin die Frage stellt, ob es bei der Erziehung Bereiche gebe, die das Elternpaar miteinander aushandeln müsse. Die Eltern berichten, dieselben Erziehungsvorstellungen zu verfolgen, abgrenzend davon 
erzählt die Mutter, dass ihr Mann in seinem Sorgehandeln bereits häufiger Maßregelungen durch andere Frauen ausgesetzt war. Als sich der Vater während des Interviews erneut über diese Situationen, in denen er als unwissender Vater adressiert wurde, ärgert, gibt die Mutter dem Gespräch eine neue Richtung.

Fr. T.: Ja Frauen fühlen sich schon ein bisschen oft auf den Schlips getreten, wenn wenn einfach Männer auch (ein Wort unverständlich: daheim?) den Weg da gehen sozusagen. Es ist, ich bin da anders. Mich stört's wirklich nicht. Im Gegenteil, es ist mir sogar sehr wichtig, ich muss mich da gar nicht so viel zurücknehmen. Mhm man muss sagen halt einfach manchmal ein bisschen mehr lächeln über gewisse Dinge, wenn's zum Beispiel Männer schaffen es ja, das ist ein@Phänomen@((Lachen)), dass man Kleidung praktisch anzieht sozusagen. Weil's bei den Kindern und Babykleidungen ist ja voll oft so, dass hinten zugebunden wird oder so und das hat man ja selber nicht, weil hinten kann man ja sehr schlecht was zusammen machen. Also das ist bei den Kleidern aber so. Bei den üblichen Männerkleidungen macht man halt vorn zu und darum müssen auch sämtliche Knöpfe auch. Wenn da vorn normalerweise eine große Blume auf dem Rock drauf ist, die ist dann halt hinten, weil vorn die Knöpfe sind, ((Interviewerin lacht)) also das schaffen nur Männer.

(Interview Herr und Frau Terland, S. 14f., Z. 678-688)

Mit der Redensart , auf den Schlips treten ‘ - als Synonym für das Gefühl vieler Frauen beim väterlichen Engagement - wird in der Thematisierung der Mutter das Bild eines Geschlechterverhältnisses aufgerufen, in dem „Mütter sich schwer damit tun, dem Mann Raum für sein Vatersein zu geben" (Jurczyk/ Lange 2009: 2) und in dem zugleich die Sorgearbeit maternalisiert wird. Von dieser Subjektposition grenzt sich die Mutter jedoch scharf ab, indem sie ihr die Position der auf Egalität bedachten Mutter entgegensetzt und diese für sich reklamiert. Darauffolgend beschreibt sie sich jedoch als genaue Beobachterin und Kommentatorin des väterlichen Tuns. Mit der Bemerkung, über seine Fehler ,lächelnd' hinwegzusehen, wird sie in der Thematisierung als Gatekeeperin des väterlichen Engagements entworfen, die dem Vater einen gewissen Handlungsspielraum eröffnet. Mit der Darstellung dieser Differenz - der Vater ist der Ausgelachte, die Mutter die (Aus-)Lachende - wird das Engagement des Vaters deutlich abgewertet. Auf verbaler Ebene wird jedoch Toleranz hinsichtlich der Unwissenheit des Vaters demonstriert und dies im Anschluss exemplarisch am Beispiel der Ankleidesituation illustriert. Väter, so lautet ihre stereotypisierende Einschätzung, zögen Kinder eher nach praktischen Gesichtspunkten an. Indem dies generalisierend allen Männern zugeschrieben wird, werden in der Darstellung erneut Differenzkategorien aktiviert: Den kompetenten sorgenden Müttern werden die inkompetenten sorgenden Vätern gegenübergestellt. Diese, so die Darstellung, seien nicht nur wenig bewandert in der täglichen Praxis der Kinderbekleidung, sondern würden auch einfache an der Kleidung angebrachte Hinweise nicht erkennen. Umgehend wehrt sich der Vater gegen diese Zuschreibung. 
Hr. T.: Ja aber es liegt achtzig Prozent ((Fr. T. lacht)) von der Zeit am Rücken und da drücken die Knöpfe.

Fr. T.: @Naja, das läuft schon, das Kind. Das läuft schon, das Kind@.

Hr. T.: Da drücken die Knöpfe.

Fr. T.: @Ist ja ganz egal@. Aber wie gesagt, das hab @ich überall entdeckt@, quer durch alle Bildungsschichten. Also das ist ((Lachen)) und da muss man schmunzeln darüber, nicht und da darf man halt nicht ((Lachen))

Hr. T.: Ja, wer sagt das, dass das so sein muss, oder?

Fr. T.: @Ja, dass die Blume am Rücken ist? Nein, ist ja wurscht@. ((Lachen))

I.: @Deswegen sind ja extra noch mal die Schildchen drin, damit man sich orientieren kann@.

Hr. T.: Ja, die schneiden wir raus. ((gemeinsames Lachen))

Fr. T.: Ja von meiner Nichte gibt's ein Kindergartenfoto. Die hat @den Kragen da oben [zeigt auf die Nasenspitze, Anm. R.S.] und die Blumen da hinten und das ist herrlich. Aber das ist überall und jede und alle Väter machen das so. Engagierte Väter@ natürlich, die ihre Kinder anziehen und sowas find ich@super@. ((Lachen))

I.: @Aber das sind ja auch Nettigkeiten, irgendwo@.

Fr. T.: Ja es ist eh herrlich. Und so muss man halt dem Ganzen irgendwie begegnen und manchmal sind halt andere Dinge, die schon ein bisschen, wo man sich dann vielleicht als Mutter denkt, okay ja mhm ja da ist vielleicht der Zugang einfach ein bisschen anders und da muss man halt einmal, reden wir halt mal drüber und und meistens ist es dann eh so, dass man irgendwo einen Weg findet, dass dir irgendwas einleuchtet, oder mir irgendwas einleuchtet. Das hat dann eh gepasst.

(Interview Herr und Frau Terland, S. 15, Z. 690-722)

Der Vater weist die Adressierung als inkompetenter Vater zurück und verteidigt sein Engagement als stärker auf die kindlichen Bedürfnisse ausgerichtet. Damit wird die väterliche Ankleidungsvariante legitimiert und der Lächerlichkeit entzogen. Gerade durch den Vorzug der Funktionalität der Ankleideweise gegenüber der Ästhetik wird zwar die Differenzierung zwischen Mutter und Vater aufrechterhalten, jedoch für den Vater positiv gewendet: Er kümmere sich gerade nicht um den rein modischen Aspekt, sondern vielmehr um die Befindlichkeiten des Kindes, womit er für sich selbst die Position des sorgenden Vaters reklamiert. Die Mutter widerspricht, wenn auch in humorvoller Weise, der Selbstdarstellung dieser Subjektposition und führt seine Argumentation ad absurdum - seinen Widerstand ,fegt' ${ }^{`}$ sie gewissermaßen vom Tisch, indem sie die Diskussion (vorerst) für erledigt erklärt. Dann führt sie jedoch ihre Meinung zur Geschlechterdifferenz zwischen Müttern und Vätern weiter aus und erklärt dies als deutlich wirksamer als ,Bildungsdifferenzen“. Sie restabilisiert die Subjektposition der kompetenten, dem Vater überlegenen Mutter, indem sie sich als Beobachterin und zurückhaltende Kommentatorin des väterlichen Sorgeverhaltens entwirft. Das folgende kurze verbale Ringen um die Positionierung der Mutter als Expertin wird von ihr entschieden, indem sie die Thematik erneut beendet. An dieser Stelle mischt sich jedoch die Interviewerin ein und führt das Thema weiter, indem sie den Vater in ironischer Weise mit den Bekleidungsnormen konfrontiert, womit er erneut als unwissender Vater entworfen wird. Mit seiner Antwort opponiert der Vater gegen das 
von der Interviewerin indirekt angedeutete Frauenbündnis und glättet die PaarDifferenz: Auch wenn das Paar sich auf der interaktiven Ebene des Interviews in bestimmten Punkten uneinig ist, ist es der Interviewerin offenbar nicht erlaubt, diese Differenzierung fortzuführen. In der sich daran anschließenden Darstellung der Mutter festigt diese die Subjektposition der Gatekeeperin: Zwar wird das Engagement der Väter gelobt, die Mutter nimmt sich jedoch heraus, zu entscheiden, ob und wie weit sie das Verhalten des Vaters akzeptiert und mitträgt oder ob sie interveniert. Sie stellt sich damit auch als ,Evaluatorin“ (Meuser 2011: 77) der väterlichen Sorgearbeit dar: Dem Vater wird damit gerade kein fraglos anerkannter Platz in der Sorgearbeit zugestanden, während sich für die Mutter eine deutliche Verteidigung ihrer ,Hoheitsrechte` und ihrer Deutungsmacht konstatieren lässt.

\section{Schluss: (Un-)Ordnungen der Sorge}

Der Beitrag ging von der These aus, dass sich die Figur des aktiven Vaters, die in aktuellen Vaterschafts- und Elternschaftsdiskursen eine große Rolle spielt, in spezifischer Weise auch in den Interviews mit Eltern zeigt und gerade nicht nur Väter ,anruft', sich als solche zu zeigen, sondern auch Mütter zur Stellungnahme herausfordert. Die Häufigkeit der Darstellungen in den Interviews sowie die vehementen Versuche der Interviewpartnerinnen, die Interviewerin zu überzeugen, zeigen, dass keine Zweifel bei der Interviewerin aufkommen sollen, dass es sich bei dem betreffenden Vater nicht um einen ,aktiven Vater handeln könnte. Es entsteht somit der Eindruck, dass diese Diskurse nicht nur für die Väter Anrufungen zur , aktiven Vaterschaft' enthalten, sondern letztendlich auch für die Mütter, sich als Teil eines egalitären Elternpaares zu zeigen und damit auch den Vater ihres Kindes/ihrer Kinder als aktiven Vater zu präsentieren. Damit erhöhen solche Diskurse gerade nicht nur den normativen Druck auf Väter, wie es Meuser (2009: 85) konstatiert, sondern, so muss ergänzt werden, offenbar auch auf die Mütter. Die Mütter zeigen sich jedoch eigensinnig und widerspenstig in der Beantwortung der Diskursanrufungen: Zwar weisen sie die Väter als engagierte aus, transformieren die Figur des ,aktiven Vaters' jedoch zugleich in die des ,inkompetenten, anleitungsbedürftigen Vaters'. Sie entwerfen sich damit vielfach als ,Zugangskontrolleurinnen' des Sorgeterritoriums, die über die Möglichkeiten der Väter entscheiden, in den familiären Bereichen aktiv zu werden. In den Darstellungen zeichnen sich damit weiterhin Reste der alten Geschlechterasymmetrie in der Elternschaft ab, indem Mütter die Hauptverantwortung und Sorgehoheit beanspruchen und der Vater als unterlegener Neuling behandelt wird. 
Dies, so die abschließende These, hängt nicht zuletzt damit zusammen, dass die Diskurse zur egalitären Elternschaft zwar den Vater als gleichgestellten Kooperationspartner anrufen, jedoch vielfach Mütter auch weiterhin wenn auch manchmal eher subtil - als Hauptverantwortliche für die gute Entwicklung des Kindes adressieren (vgl. Schneider 2002: 12). ${ }^{7}$ Studien zur Arbeitsteilung in Familien (vgl. u.a. Cornelißen 2005: 267; Cornelißen/Rusconi/ Becker 2011: 14) zeigen, dass der diskursiv vielfältig verhandelten und immer wieder thematisierten Figur des aktiven Vaters - die ja immer wieder als Synonym für eine egalitärere Arbeitsverteilung gilt - oftmals auch weiterhin die ungleiche Verteilung der täglich geleisteten Sorgearbeit gegenübersteht und sich damit offenbar für Mütter in Bezug auf die Sorgearbeit wenig verändert. Transformationen der Sorge finden derzeit offenbar noch eher auf diskursiver Ebene denn auf der Ebene familialer Praxis statt. Zudem ist die Figur des , aktiven Vaters' sehr positiv aufgeladen und bietet Vätern auch neue Anerkennungsfelder, während für Frauen im Rahmen der Sorgearbeit das Pendant fehlt. „Auf der Ebene der Politik, auch und gerade der derzeitigen Familienpolitik und der Arbeitsmarktpolitik, sind, gute Mütter vor allem solche, die einen ,guten Beruf' haben, ,gutes Geld“ nach Hause bringen, für , gute (materielle) Verhältnisse' sorgen. Zugleich sind ,gute' Mütter im Sinne des kleinkindlichen Bildungswesens oder der Jugendhilfe solche, die für ihre Kinder dauerverfügbar sind und allerlei zeitliches, finanzielles und inhaltliches Engagement zugunsten der Bildung der Kinder zeigen“" (Thiessen/Villa 2009: 13). Die Ausbalancierung zwischen diesen unterschiedlichen Adressierungen - als ,traditionelle Hausfrau', weil sie ausschließlich für das Kind da sein möchte und nicht oder nur sehr wenig erwerbstätig ist, und der Rolle als ,Rabenmutter', weil sie genau das Gegenteil tut - muss jede Frau individuell leisten. Anzunehmen ist, dass diese Balance mehr als schwierig zu halten ist und zugleich von dem neuen „Helden“(Pohl 206: 171) - dem gesellschaftlichen, diskursiven Entwurf des aktiven Vaters, der beides vereint - empfindlich gestört wird. Die gezeigten mütterlichen Umgangspraktiken mit der diskursiven Figur des aktiven Vaters lassen sich auch als Distinktions- und Exklusionspraktiken verstehen, die sich vermutlich genau in diesem Dilemma der Mütter verorten lassen: Sie können als Versuch verstanden werden, auch weiterhin die ,Regie' in der elterlichen Sorgearbeit führen zu können und die Sorgehoheit zu behalten, da der , aktive Vater' zumindest diskursiv zunehmend Raum greift, sich jedoch noch nicht zwangsläufig Veränderungen in den Elternschaftsrealitäten zeigen.

7 Diese pränatalen Anrufungen von Müttern und Vätern sind auch Thema des von Lotte Rose geleiteten HMWK-Projekts „Statuspassage Elternschaft. Zur Herstellung geschlechtsspezifischer Ungleichheiten in den pränatalen Praxen von Müttern und Vätern“, das derzeit an der FH Frankfurt am Main durchgeführt wird. In diesem Projekt werden Institutionen der Natalität, wie Informationsabende in Entbindungskliniken und Geburtsvorbereitungskurse, ethnographisch beobachtet und die jeweiligen institutionellen Anrufungen, aber auch die Konstruktionspraxen von Mutter- und Vaterschaft praxisanalytisch untersucht. 
(Un-)Ordnungen der familialen Sorge?

\section{Literatur}

Althusser, Louis (1977): Ideologie und ideologische Staatsapparate. Aufsätze zur marxistischen Theorie. Positionen, 3. Hamburg: Verlag für das Studium der Arbeiterbewegung.

Baader, Meike Sophia (2006): Vaterschaft im Spannungsverhältnis zwischen alter Ernährerrolle, neuen Erwartungen und Männlichkeitsstereotypen. Die Thematisierung von Vaterschaft in aktuellen Print-Medien. In: Bereswill, Mechthild / Scheiwe, Kirsten / Wolde, Anja (Hrsg.): Vaterschaft im Wandel. Multidisziplinäre Analysen und Perspektiven aus geschlechtertheoretischer Sicht. Weinheim: Juventa, S. 117-136.

Butler, Judith (1997): Körper von Gewicht. Die diskursiven Grenzen des Geschlechts. Frankfurt a. M.: Suhrkamp.

Cornelißen, Waltraud (2005): Gender-Datenreport. Kommentierter Datenreport zur Gleichstellung von Frauen und Männern in der Bundesrepublik Deutschland im Auftrag des BMFSF. München: o.V.

Cornelißen, Waltraud / Rusconi, Alessandra / Becker, Ruth (2011): Berufliche Karrieren von Frauen. Hürdenläufe in Partnerschaft und Arbeitswelt. Wiesbaden: VS.

Correll, Lena (2010): Anrufungen zur Mutterschaft. Eine wissenssoziologische Untersuchung von Kinderlosigkeit. Münster: Westfälisches Dampfboot.

Ehnis, Patrick / Beckmann, Sabine (2010): „Krabbeln lerne ich bei Mama, laufen dann bei Papa“. Zur Einbeziehung von Vätern bei Elterngeld und Elternzeit - eine kritische Betrachtung. In: Feministische Studien. Zeitschrift für interdisziplinäre Frauen- und Geschlechterforschung 28, 2, S. 313-323.

Ehnis, Patrick (2009): Väter und Erziehungszeiten. Politische, kulturelle und subjektive Bedingungen für mehr Engagement in der Familie. Sulzbach/Taunus: Helmer.

Fraser, Nancy (2009): Feminismus, Kapitalismus und die List der Geschichte. In: Blätter für deutsche und internationale Politik 8/2009, S. 43-57.

Gather, Claudia / Othmer, Regine / Senghaas-Knobloch, Eva (2013): Einleitung. In: Feministische Studien. Zeitschrift für interdisziplinäre Frauen- und Geschlechterforschung 34, 2, S. 203-207.

Gerhard, Ute (2010): Geschlechterverhältnisse im Wandel: Anforderungen unter globalem Anpassungsdruck am Beispiel fürsorglicher Praxis/Care. In: Becke, Guido / Bleses, Peter / Ritter, Wolfgang / Schmidt, Sandra (Hrsg.): „Decent Work“: Arbeitspolitische Gestaltungsperspektive für eine globalisierte und flexibilisierte Arbeitswelt. Festschrift zur Emeritierung von Eva Senghaas-Knobloch. Wiesbaden: VS, S. 221-230.

Graefe, Stefanie (2010): Effekt, Stützpunkt, Überzähliges? Subjektivität zwischen hegemonialer Rationalität und Eigensinn. In: Angermüller, Johannes / van Dyk, Silke (Hrsg.): Diskursanalyse meets Gouvernementalitätsforschung. Perspektiven auf das Verhältnis von Subjekt, Sprache, Macht und Wissen. Frankfurt a. M.: Campus, S. 289-213.

Höhne, Thomas (2003): Die Thematische Diskursanalyse - dargestellt am Beispiel von Schulbüchern. In: Keller, Reiner / Hirseland, Andreas / Schneider, Werner / Viehöver, Willy (Hrsg.): Handbuch Sozialwissenschaftliche Diskursanalyse. Bd. 2, Forschungspraxis. Opladen: Leske und Budrich, S. 389-419. 


\section{Rhea Seehaus}

Hüning, Johannes / Peter, Corinna (2010): Der sozialpädagogische Blick auf Familie. In: Böllert, Karin / Oelkers, Nina (Hrsg.): Frauenpolitik in Familienhand? Neue Verhältnisse in Konkurrenz, Autonomie oder Kooperation. Wiesbaden: VS, S. 7998.

Jurczyk, Karin / Lange, Andreas (2009): Vom „ewigen Praktikanten“ zum „reflexiven Vater"? Eine Einführung in aktuelle Debatten um Väter. In: Jurczyk, Karin / Lange, Andreas (Hrsg.): Vaterwerden und Vatersein heute. Neue Wege - neue Chancen! Gütersloh: Bertelsmann Stiftung, S. 13-29.

Jurczyk, Karin / Thiessen, Barbara (2011): Familie und soziale Dienste. In: Heinze, Rolf G. / Evers, Adalbert / Olk, Thomas (Hrsg.): Handbuch Soziale Dienste: Wiesbaden: VS, S. 333-352.

Jurczyk, Karin / Rerrich, Maria S. (1993): Lebensführung, soziale Einbindung und die Strukturkategorie „Geschlecht“. In: Jurczyk, Karin / Rerrich, Maria S. (Hrsg.): Die Arbeit des Alltags. Beiträge zu einer Soziologie der alltäglichen Lebensführung. Freiburg i. Br.: Lambertus, S. 262-278.

Kassner, Karsten (2008): Männlichkeitskonstruktionen von „,neuen Vätern“. In: Luedtke, Jens / Baur, Nina (Hrsg.): Die soziale Konstruktion von Männlichkeit. Hegemoniale und marginalisierte Männlichkeiten in Deutschland. Opladen / Farmington Hills: Barbara Budrich, S. 141-163.

Kerschgens, Anke (2009): Die widersprüchliche Modernisierung der elterlichen Arbeitsteilung. Alltagspraxis, Deutungsmuster und Familienkonstellation in Familien mit Kleinkindern. Wiesbaden: VS.

Kortendiek, Beate (2010): Familie: Mutterschaft und Vaterschaft zwischen Traditionalisierung und Modernisierung. In: Becker, Ruth / Kortendiek, Beate (Hrsg.): Handbuch Frauen- und Geschlechterforschung. Theorie, Methoden, Empirie. 3., erweiterte und durchgesehene Auflage. Wiesbaden: VS, S. 442-453.

König, Tomke (2012): Familie heißt Arbeit teilen. Transformationen der symbolischen Geschlechterordnung. Konstanz: UVK.

König, Tomke (2008): Diskurstheorie als Werkzeugkiste: eine Analyse der Effekte diskursiver Praxen im Kontext familialer Geschlechterarrangements. http://www. ssoar.info/ssoar/View/?resid=18516 [Zugriff 12.12.2011].

Matzner, Michael (2004): Vaterschaft aus der Sicht von Vätern. Subjektive Vaterschaftskonzepte und die soziale Praxis der Vaterschaft. Wiesbaden: VS.

Meuser, Michael / Scholz, Sylka (2012): Herausgeforderte Männlichkeit. Männlichkeitskonstruktionen im Wandel von Erwerbsarbeit und Familie. In: Baader, Meike Sophia / Bilstein, Johannes / Tholen, Toni (Hrsg.): Erziehung, Bildung und Geschlecht: Männlichkeiten im Fokus der Gender-Studies. Wiesbaden: VS, S. 427444.

Meuser, Michael (2011): Die Entdeckung der „neuen Väter“. Vaterschaftspraktiken, Geschlechtsnormen und Geschlechterkonflikte. In: Hahn, Kornelia / Koppetsch, Cornelia (Hrsg.): Soziologie des Privaten. Wiesbaden: VS, S. 71-82.

Meuser, Michael (2009): Keine Zeit für Familie? - Ambivalenzen involvierter Vaterschaft. In: Heitkötter, Martina / Jurczyk, Karin / Lange, Andreas / Meier-Gräwe, Uta (Hrsg.): Zeit für Beziehungen? Zeit und Zeitpolitik für Familien. Opladen / Farmington Hills: Budrich, S. 215-231. 
Moser, Vera / Pinhard, Inga (2010): Care - Wer sorgt für wen? In: Moser, Vera / Pinhard, Inga (Hrsg.): Jahrbuch Frauen- und Geschlechterforschung in der Erziehungswissenschaft. Care - Wer sorgt für wen? Opladen / Farmington Hills: Barbara Budrich, S. 11-13.

Ott, Marion / Wrana, Daniel (2014): Adressierung. In: Wrana, Daniel / Ziem, Alexander / Reisigl, Martin / Nonhoff, Martin / Angermüller, Johannes (Hrsg.): DiskursNetz. Wörterbuch der Interdisziplinären Diskursanalyse. Frankfurt a. M.: Suhrkamp, S. 19-20.

Otto, Petra (2011): Errungenschaft mit Änderungsbedarf. In: Hebammenforum 2, S. 99-105.

Palz, Doris / Werneck, Harald / Beham, Martina (2006): Einführung: Männer zwischen Familie und Beruf. In: Palz, Doris / Werneck, Harald / Beham, Martina (Hrsg.): Aktive Vaterschaft. Männer zwischen Familie und Beruf. Gießen: Psychosozial Verlag, S. 13-27.

Pohl, Rolf (2006): Vater ist der Beste. Über die Wiedergeburt eines Helden im sozialwissenschaftlichen Familiendiskurs. In: Bereswill, Mechthild / Scheiwe, Kirsten / Wolde, Anja (Hrsg.): Vaterschaft im Wandel. A.a.O., S. 171-189.

Possinger, Johanna (2013): Vaterschaft im Spannungsfeld von Erwerbs- und Familienleben. „Neuen Vätern“ auf der Spur. Wiesbaden: VS.

Reichle, Barbara (1996): Der Traditionalisierungseffekt beim Übergang zur Elternschaft. In: Zeitschrift für Frauenforschung 96, 4, S. 70-89.

Reinwand, Vanessa-Isabelle (2012). „Nur Wickeltische in den Männer-WCs fehlen noch“ - Zur Rolle der ,neuen“ Väter im frühkindlichen Entwicklungsprozess. In: Baader, Meike Sophia / Bilstein, Johannes / Tholen, Toni (Hrsg.): Erziehung, Bildung und Geschlecht. A.a.O., S. 23-40.

Rüling, Anneli (2010): Das Stillen - Traditionalisierung der Arbeitsteilung durch naturalisierende Deutungen von Geschlecht? In: http://nbn-resolving.de/urn:nbn:de: 0168-ssoar-154587, [Zugriff 03.10.2011].

Rüling, Anneli (2007): Jenseits der Traditionalisierungsfallen. Wie Eltern sich Familien- und Erwerbsarbeit teilen. Frankfurt a. M. / New York: Campus.

Schneider, Norbert F. (2002): Elternschaft heute. Gesellschaftliche Rahmenbedingungen und individuelle Gestaltungsaufgaben - Einführende Betrachtungen. In: Matthias-Bleck, Heike / Schneider, Norbert F. (Hrsg.): Elternschaft heute. Gesellschaftliche Rahmenbedingungen und individuelle Gestaltungsaufgaben. (Zeitschrift für Familienforschung Sonderheft, 2) Opladen: Leske und Budrich, S. 921.

Seehaus, Rhea (2014): Die Sorge um das Kind. Eine Studie zu Elternverantwortung und Geschlecht. Opladen: Barbara Budrich.

Thiessen, Barbara / Villa, Paula-Irene (2009): Mütter und Väter: Diskurse - Medien Praxen. Eine Einleitung. In: Thiessen, Barbara / Villa, Paula-Irene (Hrsg.): Mütter - Väter: Diskurse, Medien, Praxen. Münster: Westfälisches Dampfboot, S. 7-21.

Toppe, Sabine (2010): Care-Ethik und Bildung - Eine neue ,Ordnung der Sorge ' im Rahmen von Ganztagsbildung? In: Moser, Vera / Pinhard, Inga (Hrsg.): Care Wer sorgt für wen? A.a.O., S. 69-86.

Walter, Wolfgang / Künzler, Jan (2002): Parentales Engagement. Mütter und Väter im Vergleich. In: Matthias-Bleck, Heike / Schneider, Norbert (Hrsg.): Elternschaft heute. A.a.O., S. 96-119. 
Rhea Seehaus

Wolde, Anja (2007): Väter im Aufbruch?: Deutungsmuster von Väterlichkeit und Männlichkeit im Kontext von Väterinitiativen. Wiesbaden: VS.

Wrana, Daniel (2006): Das Subjekt schreiben. Reflexive Praktiken und Subjektivierung in der Weiterbildung - eine Diskursanalyse. Baltmannsweiler: Schneider. 